\title{
Úber Leistenbildung der Descemeti nebst Bemerkungen zur Frage der Megalocornea und des Hydrophthalmus.
}

\author{
Yon \\ Dr. Ernst Kraupa, \\ Teplitz. \\ Mit 10 Textabbildungen.
}

Die Bedeutung der Spaltlampenmikroskopie ist dureh die praktisch wichtigen Ergebnisse der Untersuchungen $\mathrm{Köp}$ p es und Vog ts erwiesen. Eigene Untersuchungen, die sich nun bereits auf einen Zeitraum ron. fünf Jahren erstrecken, ergaben manchen Fingerzeig für die Klinik. So wies ich die Pigmentierung*) der Hornhauthinterfläche bei der Dystrophia e pithelialis (Fuchs) nach und zog den Schluß, daß es sich bei dieser Erkrankung um eine primäre Störung des Endothels handle. Seit längerer Zeit beobachtete ich die epithelialen Cysten bei der sog. rezidivierenden Erosion ${ }^{*}$ ), in welchen ich die anatomische Grundlage dieses so lästigen Leidens sehe. Ich fand jene eigenartige Anomalie der Hornhaut***) hereditär Luetischer, bei welcher im Lidspaltenbezirk die klare Hornhaut den Limbus überbrückend sichelförmig in die Sclera vordringt. Manche dieser Beobachtungen neben anderen in Köppes Werk reproduzierten fanden ihre Bestätigung durch Koeppe, der Endothelbefund bei der Hornhautdystrophie und die Epithelblasen der rezidivierenden Hornbauterosion erst kürzlich durch Vogt. Dieser Umstand veranlaßt mich, weitere Beobachtungen zu veröffentlichen, deren Deutung einige Vorsicht voraussetzt. Denn bei der Spaltlampenmikroskopie ergeht es uns heute nicht anders als jenen, welche sich zuerst mit der Ophthalmoskopie beschäftigten. Irrtümer sind hier ebenso unvermeidlich wie zu Beginn des AugenspiegeIns.

Stationäre Trübungen in Band-, Ring-, Streifen- und Netzform kommen in der Hornhaut sowohl in der Bow manschen als in der Descemetschen Membran vor. Sie sind zwar lange bekannt, doch wenig berücksichtigt und darum mehrfach neuentdeckt worden.

*) E. Kraupa, Pigmentierung der Hornhauthinterfläche bei Dystrophia epithelialis (Fuchs). Zeitschr. f. Augenheilk. 44. 1920.

**) E. Kra upa, bei Ko eppe, Intravitalmikroskopie 1920. S. 145.

***) E. Krau pa, Fehlen des Lederhautbandes in Sichelform. Klin. Monatsbl. f. Augenheilk. 1920 . 
Trübungen der Bow manschen Membran dieser Art wurden von Schirmer ${ }^{1}$, zuletzt von Pascheff ${ }^{2}$ ) klinisch und anatomisch untersucht. Namentlich der letztere gab höchst instruktive Bilder dieser auf eine Verletzung zurückzuführenden Erkrankung der Hornhaut. Risse und Einrollungen der Membran führen zu höchst eigenartigen netzförmigen Trübungen. Häufiger als solche scheinen ringförmige Trübungen vorzukommen, wie ich sie im Laufe der Kriegszeit und mehrfach auch später zu beobachten Gelegenheit hatte. Im Umkreis besonders dichter rundlicher, mitunter ektatischer Narben findet sich ein glasig durchscheinender. mehr weniger regelmäßiger Ring, dessen Genese vermutlich in der durch den Geschwürsprozeß hervorgerufenen Zerstörung der Bow manschen Membran beruht, die dann nach erfolgter Narbendehnung sich am Rande derselben einrollte und dadurch zur Ringbildung Anlaß gab. Nach Kauterisation von Infiltraten und seichter Ulcera serpentia kann man nach erfolgter Heilung ein ähnliches Bild. schon bei einfacher seitlicher Beleuchtung beobachten. Die zarte Narbe ist von einer ganz feinen, durch ihre Transparenz auffallenden ringförmigen Zone umgeben, die im durchfallenden Lichte an der Nernstspaltlampe beobachtet den Eindruck eines ringförmigen Spaltes in den oberflächlichen Schichten der Hornhaut macht.

Von diesen Faltungen der Bow ma nschen Membran sind durch ihre Lage in der Tiefe die Faltungen der Descemeti, die mitunter auf Wucherungen rupturierter Glashaut zurückzuführen sind, leicht unterscheidbar. Thre genauere anatomische Untersuchung verdanken wir $S t a ̈ h l i^{3}$ ), der die Bändertrübung hydrophthalmischer Augen studierte und Einrisse, Aufrollung der gerissenen Glashaut sowie Endothelwucherungen zu deren Überkleidung feststellte. Mit der Spaltlampe wurden sie zuerst durch $\operatorname{Vog}^{4}{ }^{4}$ ) eingehender Untersuchung unterzogen, der auf die morphologische Verschiedenheit von Falten und Rissen aufmerksam machte. Wenn man aber bedenkt, da $B$ nach den anatomischen Bildern Stählis $\left.{ }^{3}\right)$ Risse ausheilen können, und, dabei nur ein Ende des Risses als vorspringende Leiste dauernd sichtbar zu bleiben braucht, so ergeben sich mitunter intravital schwer zu definierende Bildungen, deren Sicherstellung erst durch anatomische Untersuchung möglich wäre. Hier soll vor allem auf die klinischen Momente näher eingegangen werden und durch Abbildung von Befunden das Interesse an diesen durchaus nicht so häufigen seltsamen Veränderungen der Hornhauthinterfläche geweckt werden.

I.

Die ersten klinischen Beobachtungen der „Glashautleisten“" stammen von J. Hirsehberg ${ }^{5}$ ). Dieser Autor sah in einem Auge sechs Jahre nach durchgemachter Keratitis parenchymatosa senkrecht gegen die Richtung 
der radiären Gefäße verlaufende „Lymphspalten“, breiter und weniger scharf als erstere begrenzt. Ferner fand er einen eigentümlich geradlinig verlaufenden, die Cornea diagonal durchsetzenden verästelten Streifen, der ,wohl eine stark brechende Flüssigkeit aber nicht Blut enthält, da er bei Drehung des Spiegelstieles bald dunkel, bald hell erscheint". Hiervon gab er auch eine allerdings nur skizzenhafte $\mathrm{Ab}$ bildung. Gleichartige Fälle beschrieben hierauf P. Fridenberg ${ }^{6}$ ) und Dimmer ${ }^{7}$. Fridenbergs ${ }^{6}$ ) Bild gibt in geometrischer Figur angeordnete Linien wieder, die vom Untersucher 13 Jahre nach Bestand. einer Keratitis parenchymatosa, ,tief unter der Oberfläche der Hornhaut " gefunden wurden. Fridenberg suchte diese Streifen als Fetta nsammlung in den Lymphräumen zu erklären, einer Erklärung, der Sohirmer ${ }^{1}$ ) ausdrüoklich widerspricht. Schirmer ${ }^{x}$ nahm an, daß im Falle Fridenberg die Hornhauthinterfläche sich im Stadium der Entzündung faltete und daß die dadurch hervorgerufenen Leisten durch Verklebung dauernd bestehen bleiben. Dimmer ${ }^{7}$ ) besohrieb zunächst. zwei Fälle, von denen der erste an beiden Augen graue streife nför mige Trübungen, der zweite eine Y förmige, den hintersten Schichten der Hornhaut angehörende Trübungsfigur aufwies. Dimmer nahm eine Faltenbild ung der Desce meti an, die er auf eine zeitweilige Drucksteigerung und mit dieser vorübergehende Hornhautektasie zurückführte. J. Hirsch berg ${ }^{5 b}$ ) beharrte weiterhin auf seiner Nomenklatur und brachte eine weitere Abbildung eines ,cornealen Lymphgefäßnetzes", die beste bis zum heutigen Tage. Es handelt sich um eine netzartige Verschlingung der in Frage stehenden Bildungen. Hierauf brachte Dim $m e r^{8}$ ) einen neuen Fall dieser Art. Er bezeichnet die einige Jahre vorher gefundenen Trübungen als Faltenbildung in den tiefsten Lagen der Hornhaut und von dieser verschieden folgenden Befund: konzentrisch zu einem Punkte oberhalb des Hornhautzentrums als Vittelpunkt liegen im unteren Bereiche der Hornhaut eine Anzahl halbkreisförmig angeordneter grauer Streifen, von denen zueinander stellenweise Brücken ziehen. Die halbkreisförmigen grauen Streifen sind beiderseits von zarten, wellig verlaufenden, hell glitzernden Linien begleitet. Auf Grund experimenteller Untersuchung (Betrachtung auf Objektträger aufgeklebter Glasstäbchen und Kanadabalsamfäden) kommt Dimmer ${ }^{8}$ zu dem Schlusse, daß eine wellige Beschaffenheit der Hornhauthinterfläche die Ursache der optischen Phänomene ist. Di m mer hebt die Regelmäßigkeit der Faltenbildung in diesem Falle gegenüber der früher beschriebenen hervor, bei welcher die Streifen verästelt und getrübt waren, während hier die Hornhaut fast vollkommen durchsichtig war. Eine Ektasie wurde diesmal nicht beobachtet. Dagegen hält es Di mmer für möglich, daß die Hornhaut zur Zeit der Keratitis parenchymatosa verdickt war, 
wodurch eine Vordrängung der Hintergewebsschichten nach rückwärts stattgefunden hätte. Nach Rückgang der Entzündung und damit der Verdickung konnten sich dann die hinteren Hornhautlagen in Falten legen.

Alle diese Funde schienen beinahe in Vergessenheit geraten zu sein, als $S t a ̈ h l i^{9}$ ) in zwei Fällen ,Systeme retrocornealer, durchscheinender, glasheller, sehr stark lichtbrechender, linienförmiger schmaler Leis ten" als eigene Entdeckung beschrieb (auf Stählis 3. Fall komme ich später zurück). „Diese Leisten sahen klinisch durchaus so aus wie die Glas ha utleisten der Haabschen Bändertrübung im Hydrophthalmusauge oder wie Kanadabalsamfäden, die man über die Hornhauthinterfläche gezogen hätte." Stähli bedient sich somit derselben Vergleichsmomente wie Dim mer, und lehnt Erklärungen, nach welchen sich die "Glashautleisten" als Folgen schwerer entzündlicher Vorderkammerprozesse darstellen sollten, ab, ebenso die Annahme einer der Leistenbildung vorangehenden Descemetifaltung, weil seine Krankengeschichten weder für die eine noch die andere Deutung Anlaß gaben. Er hält es für denkbar, daß das Hornhautendothel allein sich an umschriebener Stelle faltet und daß die beiden Blätter der Endothelduplikatur zwischen sich eine Glashautleiste absondern. Doch ist auch Stähli die Ursache der Faltenbildung des Endothels problematisch.

In allen diesen Fällen handelte es sich um ausgesprochene Beobachtungen von lange abgelaufener Keratitis parenchymatosa.

In Hirsch bergs ${ }^{5 a}$, b) Fällen wurden die Streifen 6 bzw. 18 Jahre, in Fridenberg $\mathrm{s}^{6}$ ) Fall 13 Jahre, in Dim mers ${ }^{7}, 8$ ) Fällen 3,1 und 11 Jahre, in Stählis ${ }^{9}$ ) Fällen 57 bzw. 3 Jahre nach durchgemachter Keratitis parenchymatosa beobachtet. Die Entstehung der Streifen wurde bisher von niemand gesehen.

Um so schwieriger ist es, Klarheit über ihre Entstehung zu gewinnen. Auch ist die Keratitis parenchymatosa zweifellos kein so häufiges Krankheitsbild, als man nach dem klinischen Prozentsatze annehmen möchte, die Beobachtungsmöglichkeit darum in einem territorial abgegrenzten Arbeitsgebiete nicht reichlich. Im Laufe von zwei Jahren sahen wir lediglich folgende beiden Bilder.

Fall 1 (Abb. 1): Keratitis parenchymatosa vor d rei Jahren an beiden Augen. Typische Lues cong., kong. Mitralstenose*). Schwester bereits vor fünf Jahren aus gleichem Anlasse hier in Behandlung gewesen. L. Auge ohne besonderen Befund. R. Auge: Etwas unter der Hornhautmitte, innerhalb gut aufgehellter Hornhaut, liegt tief unter den Hornhautgefäßen ein System hellglitzernder, parallel gelagerter, bogenförmiger Fa I t en. Bei der Untersuchung mit dem Binocularmikroskop zeigt sich an jeder Seite der Falte der glänzende Rand. Die Falten

*) cf. L. Hahn, Herz- und GefäBstörungen bei Lues congenita und luetischer Keimschädigung. I. Die Vasoneurose. Ir. Die angeb. Mitralstenose. Zentralbl. f. innere Med. H. 30 u. 41. 1921. 
stehen untereinander in Verbindung. Ihr Verlauf ist zum Hornhautzentrum radiär. Bei direkter Beleuchtung mit der Nernstspaltlampe erscheinen die Falten als grauliche Bilder, was in der Zeichnung zum Ausdruck gebracht ist.

Fall 2 (Abb. 2): 45 jährige Frau, vor 24 Jahren K. p. Kleine, stehend ovale Hornhäute*). Beiderseits dichte Hornhautnarben und Vascularisation. In der 1. Hornhaut diagonal von a. u. nach i. o. eine typische Leiste an der Hornhaut-

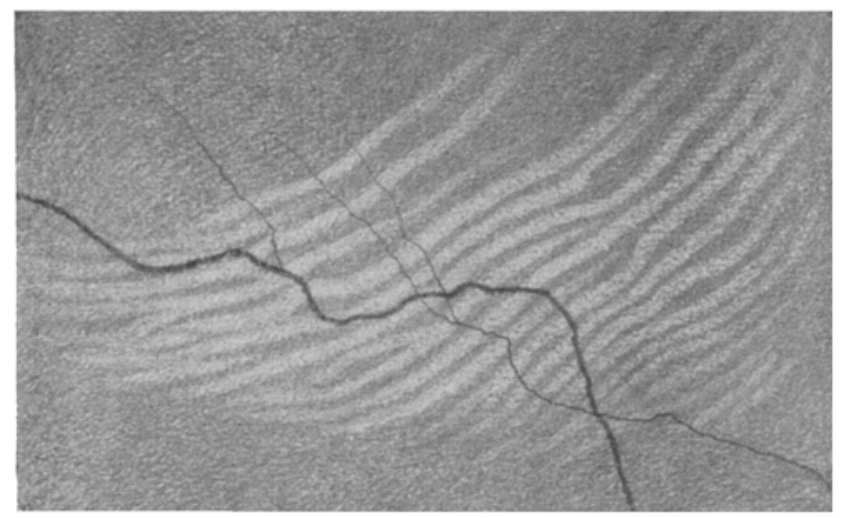

Abb. 1.

hinterfläche. Bei seitlicher Beleuchtung ist ein von zwei glitzernden Ränderm umgebener zartgrauer Streif kenntlich, der nach oben in zwei, nach unten in drei sich wieder teilende Äste ausläuft.

Die Gleichheit dieser beiden Bilder mit den aus der Literatur bekannten ist offensichtlich. Sowohl der Form als der Anordnung nach

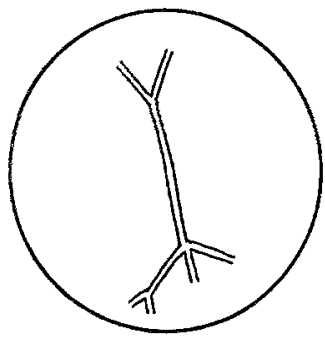

Abb, 2. sind sie mit diesen identisch. Fall 1 repräsentiert den Typus parallel gelagerter, untereinander in Verbindung stehender bogenförmiger Falten, wie er besonders durch Dim mers ${ }^{8}$ ) 3. Fall ausgeprägt ist. Fall 2 zeigt den diagonal verlaufenden ,Lymphspalt" von Hirschbergs ${ }^{5 a}$ ) Fall 1. Ein Netz solcher Bildungen, wie es in Hirsch bergs ${ }^{5 b}$ ) 2. Fall zur Darstellung kommt, ist bei Keratitis parenchymatosa bisher nicht wieder beschrieben worden. Soweit sich daher aus den fremden und eigenen Beobachtungen eine Übersicht über die Morphologie dieser Bildungen geben läßt, haben wir folgende 3 Typen zu unterscheiden:

*) Die Frage der Zugehörigkeit bestimmter Hornhautanomalien, wie der ,stehend ovalen Hornhaut" (Fuchs) und der mit "Limbusanomalien" (Kra u paWernicke) zum Komplex der ,hereditärsyphilitischen" Minderwertigkeit, läßt sich ni: ht nach serologischen Reaktionen all in, sonde $\mathbf{n}$ nur unter Mitbərücksichtigung des internen Befundes (Herz- und Gefäßsystem) lösen. 
1. parallel gelagerte miteinander in Verbindung stehende Falten [Dimmer $\left.{ }^{8}\right)$, Stähli $\left.{ }^{9}\right)$;

2. Falten in Form geometrischer Figuren [Hirschberg $\left.{ }^{5 a}\right)$, Fridenberg ${ }^{6}$ ), Dimmer ${ }^{7}$ ), Stähli $\left.{ }^{9}\right)$ ];

3. Netzbildungen [Hirschberg $\left.{ }^{5 b}\right)$ ].

II.

Seit Beginn meiner augenärztlichen Lernzeit hatte ich den beiden Bildern in Hirschbergs ${ }^{5 a, b}$ ) „Einführung“ Interesse entgegen-

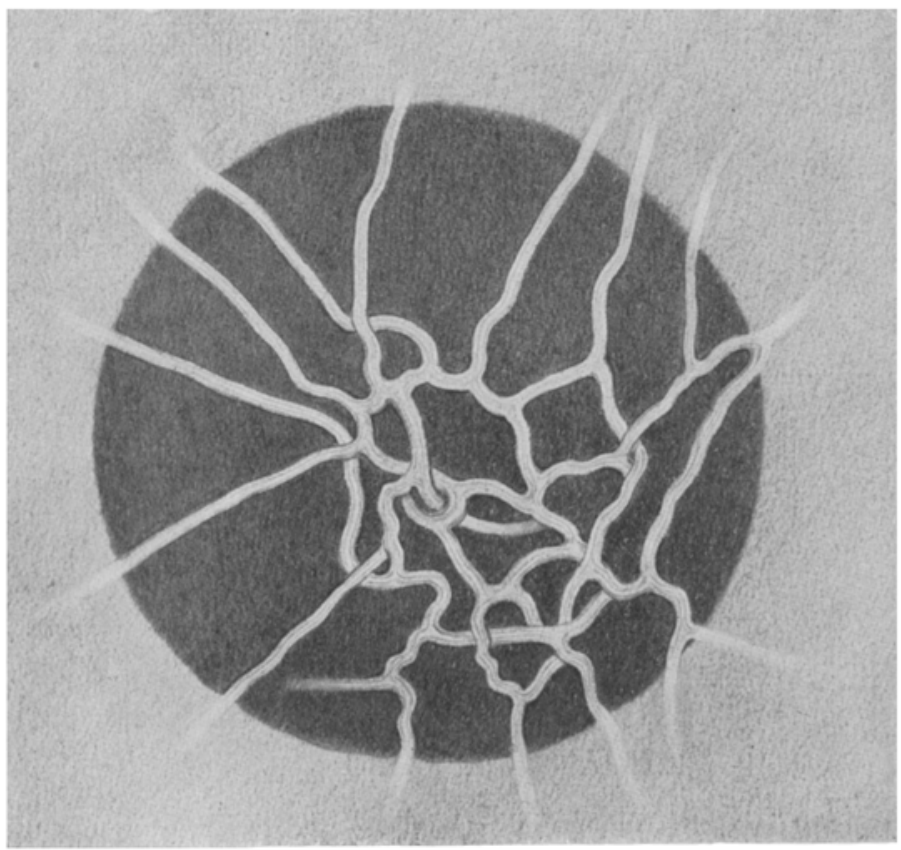

Abb. 3.

gebracht und immer wieder in einschlägigen Fällen das Rätsel dieser „L y mphspalten“ zu lösen versucht. Der Zufall brachte mir in den letzten drei Jahren neben anderen einschlägigen Bildern folgendes fast getreue Abbild von Hirschbergs zweitem Falle.

Fall 3 (Abb. 3): 60 jähriger Mann, hat vor 15 Jabren nach eigener Angabe "Herpes corneae". Stand damals durch ein Jahr in augenärztlicher Behandlung. Allerdings ist die klinische Symptomatologie der kongenitalen Mitralstenose nicht immer leicht $\mathrm{zu}$ deuten, worau mit Bestimmtheit die ungemein wichtige Arbeit von Strebel und Steiger über Korrelation von Herzfehlem und hoher Myopie hinweisen. Die Korrelation von Mitralstenose und hochgradiger Myopie erscheint nach unseren Beobachtungen feststehend, womit die Auffassung der höchstgradigen Myopie einigermaßen beeinflußt werden dürfte. 
Die Sicherung der damaligen Diagnose war zwar nicht möglich, doch gab der Augenbefund genügend Anhaltspunkte. Während die rechte Hornhaut vollständig normal war, zeigte die linke eine wenig dichte, nur im Zentrum der Hornhaut etwas stärkere Trübung mit spärlicher aiter Vascularisation und Hämosiderineinlagerung. Im Pupillargebiet zeigte sich die Hornhauthinterfläche ausgesprochen wellig gefaltet, und ein mit dem Binocularmikroskop leicht aufzulösendes Netz vorspringender Leisten wurde sichtbar. Die glitzernden Ränder der Bildungen waren auffällig und erzeugten ein ungemein zierliches Bild.

\section{Fall 4 sah ich unter meiner Beobachtung entstehen.}

Fall 4 (Abb. 4): 45 jähriger Bergmann kam mit schwerer herpetischer Erkrankung der linken Hornhaut in Beobachtung. Die Hornhaut zeigte zunächst

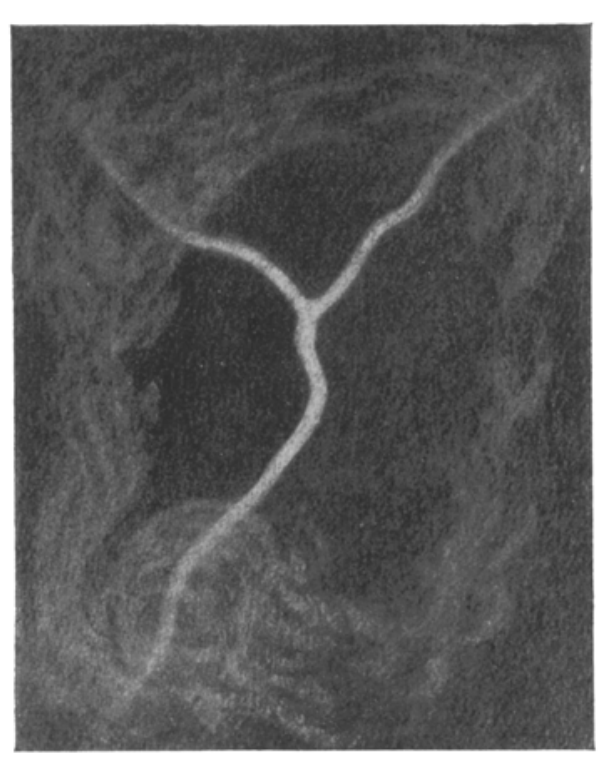

Abb. 4. einen kreisförmigen und einen Defekt unterhalb des Hornhaut. zentrums, dessen Ränder uneben fleckig infiltriert waren. (Bakteriologisch negativer Befund.) Hornhautsensibilität aufgehoben. Trotz Kauterisation schritt der Prozeß weiter, trübte die mittleren Hornhautpartien völlig und, sistierte erst nach zweimonatiger Behandlung. Bereits gegen Ende der Behandlung zeigte sich an der Hornhauthinterfläche ein vertikaler Streif von graulicher Farbe, der sich zunächst infolge der schweren Reizbarkeit des Auges nicht näher beobachten ließ. Im Laufe der späteren Monate vergrößerte er sich nach unten und begann sich nach oben Y-förmig zu teilen. Bei der nunmehr erfolgten Aufhellung der vorderen Hornhautschichten ließ sich in den tiefsten Lagen unterhalb der wolkigen herpetischen Trübungen eine den oben besshriebenen Bildungen analoge fest-

stellen. Die Form ähnelte den geometrischen Figuren der in I. beschriebenen Bildungen (Hirschberg ${ }^{5^{a}}$ ), zeigt jedoch bei näherem Zusehen eine wesentlich abgerundetere Form als jene. Die glitzernden Ränder sind sichtbar, doch nicht so ausgesprochen wie in den früheren Fällen.

Eine aus ganz unregelmäßigen Streifen zusammengesetzte Bildung sah ich in der nun 2 Jahre alten vaskularisierten und mit Krystallen durchsetzten Narben nach Herpes corneae (Fall 5).

Die einzige ähnliche Beobachtung ist in ihrer Entstehung nicht sicher geklärt. Es ist dies ein Fall Mellers ${ }^{10}$ ), in welchem sich bei sonst klarer Hornhautein Netzwerk von bandförmigen Trübungen der Hornhauthinterfläche fand, das der Anordnung nach dem von Hirschberg ${ }^{5 b}$ ) und mir (Abb. 3) wiedergegebenen außerordentlich ähnelt. Die Anamnese des Falles ist infolge der wahrscheinlich absichtlich ungenauen Angaben 
des Kranken unklar, die Krankengeschichte durch die äußeren Verhältnisse („,milit. Vormerkblatt") ungenau geführt. Legt man ihr Bedeutung bei, so hat es sich um eine herpetische Hornhauterkrankung gehandelt, wofür die verminderte Hornhautsensibilität spricht, die noch zu der Zeit bestand, da Meller den Fall sah. Berücksichtigt man, da $\beta$ zwischen der Beobachtung Mellers und dem Bestande des fraglichen „Herpes“ nur vier Monate verstrichen waren, so kann dadurch erklärt werden, daß die Entwicklung der in Frage stehenden Bildung noch nicht abgeschlossen war. So ist auch heute noch nicht in meinem Fall 4 der Glanz der Randes der Trübungsfigur so ausgesprochen wie in den übrigen Fällen.

Mutmaßlich ist hierher noch ein weiterer Fall zu zählen, den Stähli bei seinen Keratitis parenchymatosa-Fällen als Fall 3 beschreibt. Bei einem an ,Spirillenfieber" Erkrankten trat eine Hornhautentzündung des rechten Auges auf, deren Residuen Stähli ${ }^{9}$ ) nach 10 Jahren feststellen konnte. Die Aufzeichnung des Befundes unterblieb.

Jedenfalls erscheint es sicher, daß auch herpetische Hornhautentzündungen den bisher beschriebenen gleichartige „Faltentrübungen “ der Hornhaut hervorrufen können. Ihre Entstehung, die wir am Menschenauge ebenso beobachten konnten wie Sie gris t ${ }^{36}$ ) am herpesgeimpften Tierauge weist auf eine frühzeitige Beteiligung der tiefen Hornhautschichten bei diesen schweren Erkrankungen hin. Da wir aus neueren Untersuchungen über die Keratitis parenchymatosa am Menschenauge [Watanabe $\left.\left.{ }^{37}\right), \mathrm{Kunze}^{38}\right)$ ] und am Kaninchenauge [Igersheimer $\left.{ }^{39}\right)$ ] frühzeitige Veränderungen der Hornhaut an gleicher Stelle kennengelernt haben, ergibt sich hier eine eigenartige Analogie der Infektionskrankheit der Cornea. Kaum dürfte daher Di m mers Auffassung zu Recht bestehen, daß ein glaukomatöser Proze $B$ diese Hornhautveränderungen verursacht habe. Vielmehr kann umgekehrt eine Destruktion der tiefen Hornhautlagen mit und ohne Alteration der Iris ein solches Glaukom hervorrufen. Vielleicht ist ein solcher Fall von Spätglaukom bei einem Jugendlichen 5 Monate nach der Herpesheilung im reizlosen Auge so zu erklären [Kra u pa $\left.\left.\mathbf{a}^{40}\right)\right]$.

Meller lehnt in seiner Mitteilung eine traumatische Ätiologie des Herpes corneae ab. Ich glaube annehmen zu können, daß er ebenso wie die anderen Augenärzte durch die Entdeckung Grütersil) $\mathrm{zu}$ anderer Ansicht gekommen ist. Auch meine im Einverständnis mit Grüter während des Krieges fortgesetzten Beobachtungen über dieses Thema (vgl. Kra u $\mathrm{pa}^{12}$ ) haben diese ausgesprochene Übertragbarkeit besonders der dendritischen Formen des Herpes aufs Kaninchen bestätigt. Auf Grund dieser Erkenntnis ist es unmöglich, der Entstehung herpetischer gegenüber der mykotischer Hornhautgeschwüre eine Sonderstellung einzuräumen. Ebenso wie ein 
traumatischer Geschwürsprozeß durch Eitererreger infiziert zum Ulcus serpens wird, besteht die Möglichkeit, daß eine Hornhauterosion durch die fraglichen Erreger des Herpes infiziert wird. Unter diesem Gesichtspunkte sind auch ganz besondere traumatische Hornhautaffektionen zu betrachten, welche unter dem Bilde der Keratitis disciformis einhergehen. Dieser Krankheitsname ist, wie ich betont habe [Krau pa $\left.{ }^{13}\right)$ ] auch nur einer jener Sammelbegriffe, unter welchem sich eine Reihe verschiedener Krankheitsprozesse verbirgt. Sieht man ab von jenen scharfrandig begrenzten Scheibentrübungen nach reiner Kontusion, so gibt es progrediente, mit Hornhautanästhesie einhergehende Prozesse, die von einer kleinen Trübungsscheibe oder einem zunächst kleinen, später sich immer mehr vergrößernden Trübungsringe ausgehend mit schwerster Lichtscheu einhergehen und zur Ausheilung mit Narbenbildung Monate beanspruchen. Diese wahrscheinlich in das Gebiet der herpetischen Hornhauterkrankungen zu zählenden Formen waren der Anlaß zur Aufstellung des Krankheitsbildes durch Fuchs. Wir haben daher den geänderten Anschauungen Rechnung zu tragen und dort, wo herpetische Gesichtsefflorescenzen fehlen, der Anamnese des Kranken bezüglich eines Traumas Glauben zu schenken. Dies spielt namentlich dort eine Rolle, wo wie hierzulande durch den Bergwerksbetrieb zu Begutachtungstätigkeit Gelegenheit ist.

\section{IIT.}

Descemetirupturen sind beim Keratokonus klinisch zuerst von Axenfeld ${ }^{14}$ ) beschrieben worden. Mutmaßlich steht das Exacerbieren des Keratokonus mit diesen Rupturen in Zusammenhang, während die Genese des Keratokonus namentlich von jenen Autoren, welche sich mit anatomischen Untersuchungen beschäftigt haben, als von den Läsionen der Descemeti unabhängig angesehen wird, um so mehr als Fälle untersucht wurden, in welchen sich keinerlei Läsionen dieser Art fanden. Tatsächlich scheinen ausgesprochene Rupturen der Descemeti im klinischen Bilde des Keratokonus weitaus seltener, als man anzunehmen geneigt ist. Ich selbst habe seit 1914 in den zahlreichen von mir beschriebenen Fällen keinen einzigen Descemetiriß mehr gesehen. Dagegen ergibt sich bei Benützung der N.-Sp.-Lampe die Möglichkeit eigenartige ,Faltungserscheinungen" der Cornea zu studieren, die sonst bei einfacher seitlicher Beleuchtung nicht leicht sichtbar" gemacht werden können. Aus der Zeit vor Anwendung der Intravitalmikroskopie stammt eine einzige Beobachtung Elschnigs ${ }^{15}$ ) mit folgender Beschreibung: „Außerdem sieht man im Parenchym gelegen neben den intensivsten Trübungsstreifchen eine zarteste ungefähr vertikal verlaufende Schraffierung, aus feinsten graulichen Linien zusammengesetzt, die ganz den Eindruck macht, als ob eine zarteste Fältelung des Hornhautparen- 
chyms bestünde." Ähnliche Beobachtungen machten Strebel und Steiger ${ }^{30}$ ), ferner Augustein ${ }^{31}$ ).

Köppe ${ }^{16}$ ) fand ,echte" und ,unechte" Streifentrübungen beim Keratokonus, von welchen die ersteren auf Faltung, die letzteren auf Defektbildung in der Desce meti zurückzuführen seien. Köppe gibt der Ansicht Ausdruck, daß die Streifenbildung der Ausdruck bestehender oder früher einmal bestandener Progredienz sei.

Weiters beobachtete ich selbst [Kraupa $\left.\left.{ }^{17}\right)\right]$ an zwei Fällen eine zarteste streifige Trübung der Hornhaut, welche ich ins Parenchym verlegte.

In seiner kurz darauf erschienenen Arbeit beschreibt Vog t ${ }^{4}$ ) ähnliche Streifungen der Hornhaut, welche aus feinsten vertikal oder schräg vertikalen Linien zusammengesetzt wurden. Er vermutete zuerst Descemetifalten, gelangte jedoch auf Grund spätererUntersuchungen zu der Überzeugung, daß hier Veränderungen im Parenchym vorliegen. Diese geänderte Auffassung $\left.V \circ g \mathrm{ts}^{4}\right)$ trifft zweifellos für eine Reihe von Fällen zu, ganz bestimmt für meine in Wien beobachteten zwei Fälle. Die zarten Linien, die innerhalb völlig klaren Gewebes sichtbar waren, zeigten eigenartige optische, für mich

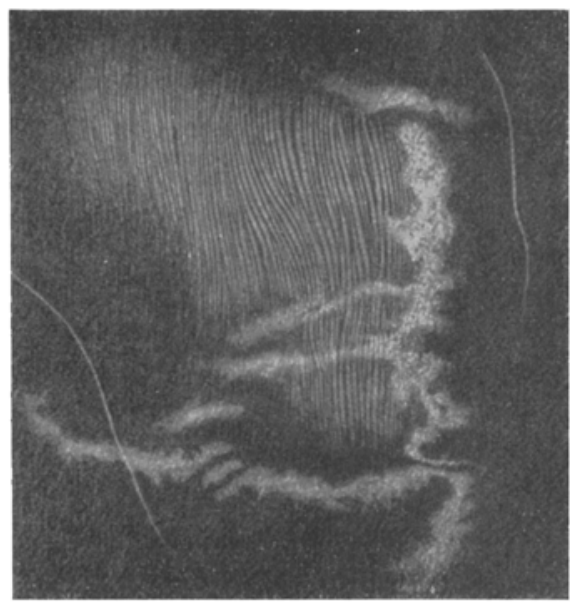

Abb. 5. nicht leicht wiederzugebende Verhältnisse. Sie machten den Eindruck, als ob verschieden brechende Substanzen in Streifenform nebeneinander gelagert wären. Das Parenchym der Keratokonushornhaut mag vor der Kegelentwicklung bereits Veränderungen der Lamellen aufweisen, die durch den Ektasierungsprozeß um so ausgesprochener zutage treten. Vielleicht haben wir es mit einer infolge der Dehnung der Hornhautbänder etwas ausgesprocheneren Streifenbildung $\mathrm{zu}$ tun, wie sie Kö $p \mathrm{pe}^{18}$ durch das Polarisationsmikroskop sichtbar gemacht hat.

Neben diesen zartesten Streifen scheint ein den tiefsten Hornhautschichten, wahrscheinlich doch der Descemeti angehörendes Faltensystem nachweisbar zu sein, wie ich es in folgendem Falle sah.

Fall 6 (Abb. 5): 28 jähriges Mädchen mit charakteristischem Gebiß der kongenitalen Lues, kongenitalen Mitralstenose und sonstigen Stigmen heredi. tärer Belastung. Beiderseits typischer Keratokonus mit Hämosiderinring. 
Am linken Auge ist im medialen Bereiche des Kegels unterhalb eigenartiger fleckiger, unregelmäBiger Parenchymtrübung ein welliges Streifensystem sichtbar, welchem die feine Transparenz der obenerwähnten Parenchymstreifung abgeht. Die Streifen sind zweifellos breiter, verschieden hoch, stellenweise dichotomisch geteilt, bei direkter Beleuchtung grauweiß. Das Glitzern der Faltenränder, wie wir es sonst beobachten, ist nicht nachzuweisen. Im ganzen erinnert die Abildung an ein daktyloskopisches Experiment. Vl Fingerzählen ohne Besserung, Vr mit $-9,0$ dptr. cyl. A. $60^{\circ} \ldots 6 / 12$.

Fall 7. 26 jähriger Mann, beiderseits klare hochgradige Hornhautkegel. Typische Lues hereditaria, kong. Mitralstenose. In der rechten Cornea die gleiche Streifenbildung wie in Fall 5. Hämosiderinring bdsts.

Jedenfalls ist weitere Beobachtung auch hier vonnöten, weil verschiedene Befunde ganz weniger Fälle vorliegen, die nicht ohne weiteres identi fiziert werden können. Neuestens behauptet Cla use $\mathrm{n}^{19}$ ) die Seltenheit des Vorkommens des Hämosiderinringes gegenüber Fleis cher. Für dergleichen feine Details ist die Benützung der Hirsch berg schen Lupe und einfacher seitlicher Beleuchtung der N.-Sp.-Lampenuntersuchung vorzuziehen, wie Fleischer und ich selbst mehrfach betont haben. Man muß dann jeden Fall so oft untersuchen, bis man des Ringes ansichtig wird, was mitunter erst nach mehrfachen vergeblichen Versuchen gelingt. Jedenfalls ist es ausgeschlossen, daß von sechs Fällen Cla usens ${ }^{19}$ ) nicht ein einziger den Ring aufwies, während Fleischer ihn fast regelmäßig, ich selbst ihn stets nachwies. Sehr interessant ist die konstit u tio nelle Eig e nart unserer beiden Fälle, die neben der von Stähli20) kürzlich erwähnten „Heredität" des Keratokonus ein eigenartiges Streiflicht auf die Pathogenese dieses Krankheitsbildes wirft. Unter diesern Gesichtspunkte erscheint es mir nicht mehr ausgeschlossen, daß Siegrists Auffassung dieses Krankheitsbildes insofern Berechtigung besitzt, als tatsächlich bei den Trägern Störungen der Drüsen mit innerer Sekretion nachgewiesen werden dürften, die allerdings nicht immer besonders ausgesprochen sein müssen. Damit ist jedoch keineswegs gesagt, daß die Entstehung des Keratokonus auf diese Störungen zurückzuführen ist, ebensowenig wie die Keratitis parenchymatosa auf innersekretorischen Alterationen beruht, welche allerdings ein ge $\mathrm{m}$ ei $\mathrm{n}$ sames Merkmal fast aller kongenital Luetischen darstellen. Wesentlich erscheint es mir daher in allen Fällen von Keratokonus, auf eine kongenitale Mitralstenose zu achten, die unter Berücksichtigung meiner früheren Befunde mit Sicherheit in der Mehrzahl der Fälle von Keratokonus sicherzustellen sein dürfte.

IV.

Ich untersuchte hierauf Vater und Tochter mit folgenden Befunden:

Vater, 41 jähriger mittelkräftiger Mann, physiognomisch ausgesprochen hereditär-luetisch, kongenitale Mitralstenose. Wassermannsche Reaktion negativ. Er ist das einzige uberlebende von 20 Kindern, die meist gleich nach der Geburt 
starben. Nur eine Schwester wurde einige Monate alt, kam mit ,weißen" Hornhäuten*) zur Welt, ohne daß die Augen eiterten. Seine Frau abortierte zweimal, erst der dritte Partus war normal. Diese : Kind entwickelte sich schlecht, hat heute im Alter von 9 Jahren das Aussehen eines Zwerges und ist geistig minderwertig, typisch heredität-luetisch, kongenitale Mitralstenose. Die Eltern hatten die Augen des Kindes nur als „Erbstück" des Vaters angesehen, weil auch dieser "eigenartige" Augen besitzt.

Fall 8. Befund des Kindes: Typischer beiderseitiger Hydrophthalmus. Große, stark gewölbte Hornhäute. Es gelang infolge der Unruhe des Kindes nur die

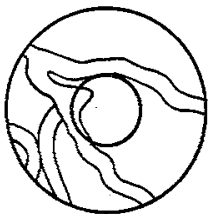

Abb. 6.

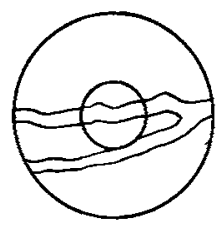

Abb. 7.

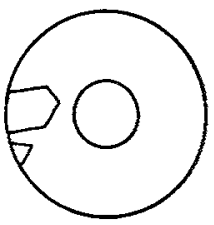

Abb. 8.

ophthalmometrischen Maße am linken Auge zu gewinnen: 6 dptr. Diff., st. br. Mer. $90^{\circ}$. Hornhautradien: 7,9 und 8,9. In beiden Hornhäuten typische Bändertrübungen (Abb. 6 u. 7). Irides auffallend dunkel. Ähnlich strukturlos wie die des Vaters, fehlende Krause. In der rechten Iris sind an der lateralen Ansatzstelle zwei Iridodiastasen (Abb. 8) sichtbar, von welchen die obere breiter, die untere kleiner und eckig geformt ist. Die Sehnerven sind gut gefärbt, weisen kleine, schüsselförmige physiologische Exkavationen auf. Tension (mit dem Finger geprüft) beiderseits sichtlich erhöht. Auf Pilocarpin Aufhellnug der sonst leicht getrübten Hornhaut. $\mathrm{V}=\mathrm{R} .0,2$ o. C. L. desgleichen.

Fall 9. Befurd des Vaters: Sclerae bläulich, in der Nähe des Limbus corneae beiderseits der Ciliarkörper durchscheinend, dessen vordere Grenze schon bei seitlicher Beleuchtung im oberen Bereiche in Form schwarzer Lappen durch die Sclera durchscheint. Mit der Sachsla m pe zeigt sich diese Lappung entsprechend deutlicher. Die Sclera geht beiderseits besonders im oberen Bereiche ohne Wölbungsunterschied in die Hornhaut über. An Stelle des Limbus comeae ist ein $\mathrm{kreidigweißer} \mathrm{Ring} \mathrm{sicht-}$ bar (Fötalring), an den sich eine breite Zone ge-

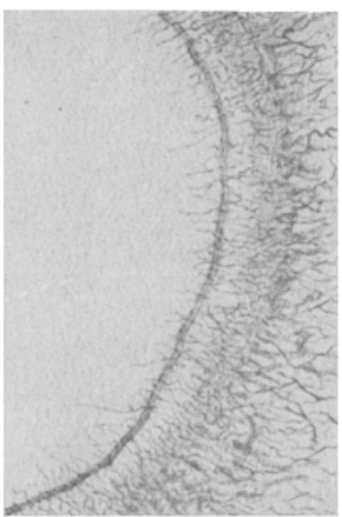

Abb. 9. trübten, unscharf in das klare Hornhautgewebe übergehenden Gewebes anschließt. Im oberen Bereiche, $1 / 2 \mathrm{~mm}$ vom Fötalringe entfernt, innerhalb der Hornhaut, sichelförmige weiBe Trübung (E mbryotoxon). Diffuse zarteste parenchymatöse Trübungen. Hornhäute klein: D. M. vert. $10 \mathrm{~mm}$, horiz. $11 \mathrm{~mm}$. Javal r. Auge $51 / 2$ dptr. Diff. st. br. Mer. $170^{\circ}$. Hornhautradien: 7,5 und $8,5 \mathrm{~mm}$. 1. Auge 2,0 dptr. Diff. st. br. Mer. $170^{\circ}$. Hornhautradien: 7,8 und $8,0 \mathrm{~mm}$. An der Hornhauthinterfläche liegt knapp vor dem „Fötalringe“ eine bei Nernst. spaltlampen-Beleuchtung deutlich vortretende Descemetileiste, die die Hornhaut umkreist $(\mathrm{Abb}$. 9). Sie ist schwer sichtbar zu machen, zeigt wenig ausgesprochenen Glanz and viel aufgelagertes dunkles Pigment. Bei entsprechender

*) Vermutlich ,kongenitale Hornhauttrübung". 
Beleuchtung wird der Eindruck erweckt, als bilde die Falte die Grenze einer Zunge trüben Gewebes, welche in die Hornhaut vorspringt. Die Vk. tief, leichtes Irisschlottern. Irides dunkel, auffällig substanzarm. Krause fehlt. Der Ciliarteil ist bräunlich, der Sphincterteil graulich gefärbt. Bei stärkerer Mikroskopvergrößerung sieht man ein spärliches Trabekelwerk, das im Sphincterteil auf einer graulichen, im Ciliarteil einer mehr bräunlichen Unterlage ruht. Keine Krypten. Mit der Sachs lampe: Ciliarteil netzartig durchleuchtbar. Pupillen: r, rund, I. etwas eckig, beide auf Licht und Konvergenz normal reagierend. In der linken Iris findet sich im oberen Bereiche knapp bis an den Sphincterteil heranreichender dreieckiger Irisausschnitt (Iridodiastase), dessen medialer und lateraler Rand je einen kleinen Gewebsanhang tragen (Abb. 10). Die Breite des Dəfektes

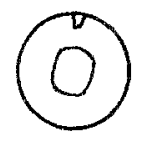

Abb. 10 . an der Wurzel $1^{1 / 2} \mathrm{~mm}$. Dort Linsenrand sichtbar. Pupillarmembranreste, ebensolche auch auf der vorderen Linsenkapsel. In der Linse k]einste fleckige Trübungen wie sonst bei kongenitalen, punkt. förmigen Katarakten. O phthal moskopisch: Papillen normal mit flachen, schüsselförmigen Exkavationen, schmalem Skleralring, temporal und anschließend daran besonders links unregelmäßige, lappige Aderhautatrophie. Fundus stark getäfelt.

V. R. $-3,0$ dptr. sph. c- $-5,5$ dptr. eyl. A. $70^{\circ} \ldots$. . 0,5.

L. mit $-10,0$ dptr. sph. ... 0,4 .

Schiötz R. L. 18.

Die Beurteilung der klinischen Details dieser beiden Fälle erforderte die Aufrollung der verschiedensten Fragen, deren einigermaßen erschöpfende Behandlung den Rahmen dieser Mitteilung weit überschreiten würde. Ich will mich daher auf die Besprechung der wichtigeren Momente beschränken, ohne die Literatur besonders zu zitieren.

Es interessiert hier vor allem die Descemetiveränderung der Hornhaut des Vaters, während die des Kindes ohne weiteres der wohlbekannten Haabschen Bändertrübung zuzuzählen ist. Beim Vater bestand eine eigenartige circulär verlaufende einzelne Descemetileiste. Stähli ${ }^{3}$ ) erwähnt eine hinter d enLimbus corneae ziehende bogenförmige Descemetileiste und jüngst $G$ u ist ${ }^{21}$ ) eine ähnliche mehr zentral gelegene circulär verlaufende Bändertrübung, hier aber handelt es sich um eine einzelne, die ganze Hornhaut umkreisende Leiste. Prinzipiell dürfte es sich um gleichartige Bildungen handeln, wie dies Stähli') bezüglich der Leisten bei der Keratitis parenchymatosa betont hat. Die Genese der Leiste* in unserem Falle ist jedoch sehr schwer erklärbar, gar wenn man die widerspruchsvollen Auffassungen des Verhältnisses des $\mathrm{Hy}^{-}$ drophthalmus zur ,Megalocornea“s in Betracht zieht.

Es handelt sich in unserem Falle scheinbar um eine Mikrocornea im Sinne Stählis ${ }^{22}$ ), deren Breitenmaße zu groß angeführt sind, weil

*) Vielleicht handelt es sich um jene von Axenfeld als „Embryotoxon posterius" bezeichnete Bildung. (Heidelb. ophthalmol. Ges. 1920.) Bei der von mir beobachteten "Hornhautanomalie " gibt es zwei Formen: a) Herübergreifen klarer Hornhaut über die Sclera; b) sichelförmiges Vordringen der Sclera in die tiefen Hornhautschichten. Im ersten Falle ist das Gebiet der Cornea vergrößert, in zweiten eingeengt. 
sie von der trüben Umrandungszone aus gemessen sind. Dagegen sind die Hornhautradien weitaus größer als die der Stählischen Fälle, welche fast immer kleine Hornhautradien bei hoher Hornhautrefraktion darboten. Somit entsprechen die hier ermittelten Zahlen großen flachen Hornhäuten, wie wir sie beim Hydrophthalmus zu treffen gewohnt sind. Es ließe sich daher behaupten, daß es sich um einen in frühem Stadium ausgeheilten Hydrophthalmus [vgl. Salzmann $\left.\left.{ }^{23}\right)\right]$ handelt, wenn es nicht im Wesen des „Hydr. ophthalmus" läge, daß er eine auffallend große Hornhaut, den „Keratoglobus" besitzt. Im Wege steht uns nur die Gepflogenheit, bei der Deutung unklarer Krankheitsbilder sich an die Extreme zu halten und diese als scharf umgrenzte Typen der Systematik nutzbar zu machen, ohne die Zwischenstufen zu berücksichtigen. Auf der anderen Seite versucht in gewiß höchst verdienstlicher Weise die moderne Richtung der Variabilitäts- und Vererbungslehre der Systematik entgegenzuwirken, bemächtigt sich aber nur zu gerne sichtlich außerhalb der Varianten stehender Typen, die ihre Entstehung entweder Krankheit [vgl.Stähli ${ }^{20}$ ] oder der Mißbildung oder auch beider verdanken können. Versuchen wir es, uns wieder von der Anschauung zu befreien, daß der Hydrophthalmus und die "Megalocornea" bzw. der „Megalophthalmus" wesensverschiedene Bildungen sind, von denen die eine lediglich auf Glaukom, die andere auf Mißbildung zurückzuführen sei, versuchen wir es, jene Form von Megalocornea, die mit totaler Vergrößerung des Augapfels einhergeht (Megalophthalmus oder Gigantophthalmus) von der ihr in der Variabilitätskurve zugewiesenen Stelle zu entfernen und dort nur jene Fälle zu belassen, welche lediglich eine relativ zu große Hornhaut aufweisen [Kestenbaum ${ }^{24}$ )], so können wir mit Fug und Recht - zumindest mit dem gleichen, mit welchem die eben erwähnten Auffassungen gezeitigt worden sind - sagen, daß Hydrophthalmus und Megalophthalmus Mißbildungen im Sinne eines Riesenwuchses des Auges sind, welche nach bestimmten Gesetzen vererbbar sind. Sind mit dem Megalophthalmus andere Anomalien des Augapfels (Fehlen des Schlemmschen Kanals, kongenitale Anomalien im Irisgewebe [Meller $\left.{ }^{25}\right)$ ] korrelativ verknüpft, so entsteht Glaukom, welches zu sekundären Veränderungen (weiterer Vergrößerung, Bändertrübung*) usw.) Anlaß gibt. Berücksichtigen wir die Arbeiten von Peters und von Hippel über die angeborene Hornhauttrübung und die hierbei vorkommende Hydrophthalmusbildung, und gehen auf eine der ersten Beobachtungen auf diesem Gebiete, die von A m mon's in seinem Tafelwerke zurück, so ist es wohl am Platze, hier auf die Anschauungen $\nabla$. Szilys ${ }^{26}$ ) von den idiogenen und peristatisch bedingten Miß-

*) Wenn die Bändertrübung in irgendeiner Form nicht bereits vor dem Glaukom existiert. 
bildungen zurückzugreifen. Wir hätten dann im Megalophthalmusauge den Typus einer idiogenen, im Hydrophthalmus eine peristatisch bedingte Mißbildung vor uns, welch letzterer je nach der Tragweite der vorliegenden Keimschädigung eine größere Variationsbreite zukommt als dem Megalophthalmus, der sich gesetzmäBig innerhalb einer Familie vererbt, ohne daß sich je Glaukom zeigt, es sei denn ein sekundäres. Dann kommt es auch nicht mehr darauf an, ob die Cornea eines $\mathrm{Hy}$ drophthalmischen groß oder klein ist, wenn nur der übrige Augenbefund auf die Zugehörigkeit zu dieser auf Mischung von Mißbildung und Krankheit beruhenden Anomalie hinweist.

Ähnliches scheint mir für die My o piefrag e zu gelten. Man unterscheidet heute nicht mehr hohe und niedere Grade von Myopie nach dem Refraktionszustande, ohne sich um Achsenlänge und Hornhautrefraktion zu kümmern. Berücksichtigt man aber noch den Befund des Augenhintergrundes, so ergibt sich mitunter die Notwendigkeit, z. B. ein Auge von 3 dptr. Myopie der deletären myopischen Mi ßbildung, wenn wir uns so ausdrücken wollen, zuzuzählen, ein Auge mit 15 dptr. dagegen ohne weiteres der Variabilitätskurve Stei gers einzufügen.

Tatsächlich zeigen die oben beschriebenen Augen von Vater und Kind außerordentlich viel Wesensverwandtes. Beider Hornhäute sind. verhältnismäßig fla ch, bei beiden bestehen Veränderungen der M. Descemeti, die Kammern sind tief. Beider Irides sehlottern und weisen Strukturanomalien auf, die auf ein Zurückbleiben a uf niederer Entwioklungsstufe hindeuten*).

Eigenartig ist auch die Vererbung der Lückeniris (Iridodiastase Ammons). Diese bei dem Vater im oberen Bereiche der Iris, bei dem Kinde im seitlichen Bereiche in Zweizahl vorkommende Mißbildung entspricht der Form nach den jüngst von Berg meister ${ }^{27}$ ) und B a y er ${ }^{28}$ ) gegebenen Befunden. Traumen sind als Ursache auszuschließen. Auch entspricht die Form dieser Bildung ganz und gar nicht dem einer traumatischen Irisablösung. Selbst bei der kleinsten peripheren Irisabreißung entsteht ein länglicher peripherer Spalt, nie ein radiärer zentral bogenförmig begrenzter Sohlitz***).

Der Bestand dieser atypischen Irismißbildung in meinen beiden Fällen, deren Genese bisher nicht geklärt ist, bestärkt mich darin, eine Keimschädigung für die Entstehung der Mißbildung verantwortlich zu machen, welche in der vererbten Syphilis von Vater und. Tochter zu suchen ist [cf. F u chs ${ }^{41}$ )]. Der negative Ausfall der Wassermannschen

*) Vgl, die Abbildung der Iris in v. Ammons Fall.

**) Wer nur den Vater zu begutachten gehabt hätte, konnte in der Diastasenbildung einen Heilfaktor für das iuberstandene Glaukom des Vaters sehen. 
Reaktion, welcher bei älteren Individuen der II., gar bei Individuen der III. Generation selbstverständlich ist, kann uns nicht über die objektiv wahrnehmbaren äußerlichen Stigmen insbesondere über die Herzanomalie im Sinne der von uns fast stets bei kongenitaler Lues gefundenen kongenitalen Mitralstenose wegtäuschen (D urozier 1877, L. Hahn).

Ich hätte noch zu erweisen, daß beim Vater einmal in früher Jugend Glaukom bestand*). Hierfür kann die diasclerale Durchleuchtbarkeit des vorderen Bulbusabschnittes und die Descemetileiste sprechen. Daß derzeit kein Glaukom besteht, beweist die mit dem Tonometer erwiesene normale Tension. Bezüglich der Zeit und Art des „Ausheilens" des Glaukoms und der Ursache dieser Erscheinung weiß ich nichts anzugeben, weil ich hierüber bisher über keinerlei Erfahrung verfüge.

[Nachschrift bei der Korrektur. Außerordentlich interessante Ausblicke ergeben sich unter Berücksichtigung der Arbeiten von $\mathrm{R} \ddot{u}-$ be $1^{32}$ ) und Fried ${ }^{33}$ ), sowie des von letzterem angefübrten Falles von Ka yser ${ }^{34}$ ). Mit der von den genannten Autoren als „Cor nea plana“ bezeichneten Hornhautmißbildung ist die oben abgehandelte Hornhautverbildung des Vaters als identisch zu bezeichnen. Dem Grade der Ausbildung nach entspricht sie am ehesten dem Falle von Kayser. Nach Friede würde sie den reinen Typus „Pse udo mikrocornea“ darstellen, doch lehne ich es ab ,Typen" anzuerkennen, wo fließender Übergang bei sicher bestehender, doch heute noch lange nicht zu erfassender Gesetzmäßigkeit besteht.

Die nahe Verwandtschaft der Fälle steht außer Zweifel. Merkwürdig sind einige besonders zu erwähnende Umstände:

Die Irisa nomalien, die fast in allen Fällen vorkommen, die vordere Synechie in einem der Fälle $R$ übels (man vgl. die Abbildung A mmons in einem Falle von kong. Hornhauttrübung [Peters] und Hydrophthalmus), die Keratitisparenchymatosa in einem der Rübelschen Fälle und das Gla ukom, das die Patientin Friedes im Alter von 20 Jahren an einem Auge durchgemacht hat. Auch die zarten parenchymatösen Hornhauttrübungen sind fast allen Fällen gemeinsam. Die Sattelnase der Patientin Fried es ist aus seiner Abb. 1. ersichtlich. Die Photographien meiner Fälle zeigen sie nicht besser. All diese Umstände neben dem familiären Auftreten stützen meine Auffassung von peristatisch bedingten Anomalien der Hornhaut, zu welchen

*) Vielleicht sind die Spätglaukome Mikrophthalmischer (Pristley S mith, Peters, Stähli) auf eine ähnliche Ursache zurückzuführen, und nur aus besonderen Gründen in späterem Alter in Erscheinung getreten. Im übrigen besteht zwischen kongenitaler Lues und Glaukom ein viel innigerer Konnex, als man nach den Literaturangaben schließen möchte. 
die ,atypischen “ Form-, Größen- und Wölbungsanamalien zu zäblen sind (auch die symmetrische Abschrägung der Hornhaut Streiff $\mathrm{s}^{35}$ ), welche mit meiner Cornealanomalie identisch sein dürfte). Nur so läßt sich die konstitutionelle Eigenart dieser Augen, die eine Mischung von Mißbildung und Krankheit darstellt, erklären.]

Es erübrigt nur noch auf zwei Literaturstellen bei Stellwag ${ }^{29}$ ) hinzuweisen.

1. „Die Descemeti ist durch Auflagerung neugebildeter glashäutiger Substanz einer Art Hypertrophie fähig. Ich fand in einem Falle den peripheren Teil der Descemeti von wenigstens doppelter Mächtigkeit und sehr steif. Unter dem Mikroskop erschienen auf der wasserhellen Glashaut eine große Menge unregelmäßiger, größtenteils parallogrammatisch begrenzter Platten mit rauhem Rand aufgelagert. Diese Plättchen waren völlig strukturlos, von völlig homogenem Gefüge und in hohem Maße durchsichtig; ihre Ränder und Faltenfirste brachen sehr stark das Licht, kurz über ihre glashäutige Textur konnte kein Zweifel obwalten, und dieses um so weniger, als auch an einigen dieser Plättchen die im $\S 7$ erwähnten, parallel und in Abständen von $0^{\prime \prime \prime}, 0055$ ziehenden oberflächlichen Risse mit Sicherheit nachgewiesen werden konnten. Es war die Bulbushöhle des betreffenden Präparates mit Markschwamm fast ganz ausgefüllt."

Ausführlich behandelt Stellwag auch die Faltenbildung der Descemeti nach Atrophie der Hornhaut. „Es zeigt sich an ihnen am schönsten die absolute Unfähigkeit der Glashäute, einmal erlangte Flächenausdehnungen durch Schrumpfung des Gewebes auf sich selbst zu vermindern."

2. Bezüglich der ,normwidrigen Vergrößerung“ der Hornhaut sagt Stellwag: „Es gibt Individuen mit auffallend großen vorspringenden, widrig glotzenden Augen, in welchen der Durchmesser der Hornhaut die Norm um $1^{\prime \prime \prime}$ und selbst etwas mehr übertrifft. Ob die Augen als wahre Abnormität zu betrachten sind, steht dahin. Die Sehfunktion ist in ihnen nur wenig gestört, ein Merkmal, welches sie hinlänglich von den weit häufigeren und ganz sicher abnormen Vergrößerungen des Augapfels und der Hornbaut unterscheidet, bei welchen die Vergrößerung nicht sowohl auf normwidrige vermehrte Anbildung der konstituierenden Elemente als vielmehr auf eine Ausdehnung der vorhandenen und duroh krankhafte Prozesse widerstandsunfähig gewordenen Gewebe zu schreiben ist."

Hieraus geht hervor, daß Stell wag bereits eine anatomische Untersuchung der Descemetiwucherung bei einem hydrophthalmischen Gliomauge durchgeführt hat, und $\mathrm{da} \beta$ er vor Horner den gleichen Standpunkt wie dieser in der Megalocorneafrage eingenommen hat. 
nebst Bemerkungen zur Frage der Megalocornea und des Hydrophthalmus.

\section{Erklärung der Textabbildungen.}

Abb. 1, 3, 4, 5, 9. Gezeichnet mit dem Binokularmikroskop Oc. 2, Obj. $a_{2}$. Abb. 2. Skizze vergrößert.

Abb. 6, 7, 8, 10. Skizzen in natürlicher Größe.

Die Abbildungen sind die letzten meines hochbegabten Zeichners $\mathrm{H}$ an slik, der an einer im Felde erworbenen Tuberkulose in jugendlichem Alter starb.

\section{Literaturverzeichnis.}

1) Schirmer, 0., Über Faltungstrübung der Hornhaut. Arch. f. vergl. Ophthalmol. 42, III, 1. 1896. $-{ }^{2}$ ) Pasoheff, C., Anatomische Untersuchungen über die indirekten Rupturen der Membrana Bowanii mit Bemerkungen iuber die Entstehung der bänder- und netzförmigen Keratitis traumatica. Klin. Monatsbl. f. Augenheilk. 61, 678. 1918. - ${ }^{3}$ ) Stähli, J., Klinik, Anatomie und Entwicklungsmechanik der Haabschen Bändertrübungen im hydrophthalmischen Auge. Arch. f. Augenheilk. 89, 141. 1915. - ${ }^{4}$ ) Vogt, A., Reflexlinien durch Faltung spiegelnder Grenzflächen im Bereiche von Cornea, Linsenkapsel und Netzhaut. Arch. f. vergl. Ophthalmol. G. 99, 296. 1919. - $\left.{ }^{5}\right)$ Hirschberg, J., a) Ophthalmoskopie in Eulenburgs Realenzyklopädie 1888; Dtsch. med. Wochenschr. 1888, Nr. 25 u. 26; Zentralbl. f. prakt. Augenheilk. 1888, S. 259. „Ausgewählte Abhandlungen" 1913; Zentralbl. f. prakt. Augenheilk. 1895, S. 473. Ref, zu Fridenberg. b) „Einführung in die Augenheilkunde“, Bd. II, S. 101. 1901. - ${ }^{6}$ ) Friden. berg, P., A geometrical system of opaques lines in the cornea: an undescribed sequela of interstitial Keratitis. N. Y. Eye and Ear Infirmary Reports, Jan. 1895, zit. nach Schirmer. -- 7) Di m mer, F., Über Faltungstrübung der Hornhaut nach Keratitis parenchymatosa. Zeitschr. f. Augenheilk. 5, 251. 1901. —8) Dimmer, F., Tine besondere Art persistierender Hornhautveränderung (Faltenbildung) nach Keratitis parenchymatosa. Zeitschr. f. Augenheilk. 13, 635. 1905. 9) Stähli, J., Über persistente retrocorneale Glashautleisten in ehedem parenchymatosakranken Augen. Klin. Monatsbl. f. Augenheilk. 2, 336. 1919. - ${ }^{10}$ ) Meller, J., Zur Kenntnis der bänderförmigen Trübung der Hornhaut. Klin. Monatsbl. f. Augenheilk. 2, 135. 1917. - $\left.{ }^{11}\right)$ Grüter, W., Experimentelle und klinische Untersuchungen über den Herpes corneae. Dtsch. ophthalmol. Ges. Heidelberg 1920. 12) Kraupa, E., Zu Grüters ätiologisehen Untersuchungen über den Herpes fibrosa. Münch. med. Wochenschr. 1920, Nr. 42, S. 1236. - ${ }^{13}$ ) Krau pa, E., cf. L. Köppe, Die Mikroskopie des lebenden Auges. Berlin 1920. S. 159-162. - ${ }^{14}$ ) Axenfeld, Über akute Exacerbationen des Keratokonus durch Spontanruptur der Membrana Descemeti. Klin. Monatsbl. f. Augenheilk. 2, 160. 1905, - ${ }^{15}$ ) Elschnig, A., Über den Keratokonus. Klin. Monatsbl. f. Augenheilk. S. 25, 1894. - ${ }^{16}$ ) Kö ppe, L., Klinische Beobachtungen mit der Nernstspaltlampe und dem Hornhautmiksoskop IV und V. Arch. f. Ophthalmol. G. 93, H. 2, S. 210. 1917. - 17) Kraupa, E., Ein weiterer Beitrag zur Auffassung des Krankheitsbildes des „Keratokonus“ III. Zentralbl. f. prakt. Augenheilk. 1919, Mai/Juni. $\left.{ }^{18}\right)$ Köppe, L., Die Ultra- und polarisationsmikroskopische Erforschung des lebenden Auges und ihre Ergebnisse. Bern 1921. - ${ }^{19}$ ) Cla us en, Keratokous und seine Behandlung. Dtsch. ophthamol. Ges. Heidelberg 1920. S. 288. - ${ }^{20}$ ) Stähli, J., Das Krankheitsbild des Keratokonus vom Standpunkte der Variabilitätslehre. Klin. Monatsbl. f. Augenheilk. 1, 712. 1919. - ${ }^{21}$ ) Guist, G., Ein Beitrag zur Klinik der Haabschen Bändertr übung. Zeitschr. f. Augenheilk. 44, 242. 1920. $\left.{ }^{22}\right)$ Stähli, J., Klinische Untersuchungen an Mikrocorneaaugen, zugleich ein Beitrag zur Megalocorneafrage. Klin. Monatsbl. f. Augenheilk, 62, 316. 1919. -

${ }^{23}$ ) Salzmann, Bearbeitung von Fuchs, Lehrbuch der Augenheilkunde 1921. - 
$\left.{ }^{24}\right)$ Kesten ba um, Über Megalocornea. Klin. Monatsbi. f. Augenheilk. 62, 733. 1919. - $\left.{ }^{25}\right)$ Meller, J., Hydrophthalmus als Folge einer Entwicklungsanomalie der Iris. Arch. f. Ophthalmol. G. 9\%, 34. 1916. - ${ }^{26}$ ) v. Szil y, Ergebnisse neuer Experimentalforschungen für die verschiedenen Formen der angeborenen Stare und ihre theoretische Bedeutung für die Mißbildungslehre. Dtsch. ophthalmol. Ges. Heidelberg 1918, - ${ }^{27}$ ) Bergmeister, Über Polykorie und verwandte seltenere Irisanomalien. Zeitschr. f. Augenheilk. 41, 82. 1919. - ${ }^{28}$ ) Bayer, E., Reichenberger Korrespbl. 1919; ref. Zentralbl. f. d. ges. Ophthalmol. _- ${ }^{29}$ ) Stell wag, Die Ophthalmologie vom naturwissensehaftlichen Standpunkte aus bearbeitet. Frlangen 1853. - ${ }^{30}$ ) Strebel und Steiger, Über Keratokonus. Klin. Nonatsbl. f. Augenheilk. 1, 260. 1913. - 31) Augstein, Zur Atiologie und Therapie des Keratokonus. Klin. Monatsbl. f. Augenheilk. 1, 417. 1913. - ${ }^{32}$ ) Rübel, Kongenitale familiäre Flachhut der Comea (Cornea plana). Klin. Monatsbl. $f$. Augenheilk. 1, 427. 1912. - ${ }^{33}$ ) Friede, R., Über kongenitale "Cornea plana" und ihr Verhältnis zur Mikrocornea. Klin. Monatsbl. f. Augenheilk, 6\%, 192. 1921. - ${ }^{34}$ ) Kayser bei Friede. - ${ }^{35}$ ) Streiff, J., Über "hochstehende Augen" und formative Korrelationen und über angeborene Abschrägung der Hornhautrundung. Klin. Monatsbl. f. Augenheilk. 6r, 145. 1921. - ${ }^{36}$ ) Siegrist, Diskussion zu Grï iter, Experimentelle und klinisehe Untersuchungen über den sog. Herpes corneae. Ophthalmol. Gesellsch. Heidelberg 1920, S. 172. - ${ }^{37}$ ) Watanabe, Path. anat. Befund bei Keratolisparenchymatosa syphilitica congenita. Klin. Monatsbl. f. Augenheilk. 52. 1914. - ${ }^{38}$ ) K unze, E., Anatomische Untersuchung eines Falles von Keratitis parenchymatosa e lue hereditaria. Graefes Arch. f. Ophthalmol. 102, 205. 1920. - ${ }^{39}$ ) I gersheimer, Neue Untersuchungen zur Syphilis des Sehapparates. Außerord. Tagung der Wien. ophthalmol. Ges. 1921. Ref. Klin. Monatsbl. f. Augenheilk. 67, 317. 1921. - $\left.{ }^{40}\right) \mathrm{Kra}$ u pa, E., Elliot und Zyklodialyse. Zentralbl. f. pr. A. 1915. - ${ }^{41}$ ) Fuchs, E., Graefes Arch. f. Ophthalmol. 94, 97. 1918. 